\title{
Mapping Functional Interaction Sites of Human Prune C-Terminal Domain by NMR Spectroscopy in Human Cell Lysates
}

\author{
Donatella Diana, ${ }^{[\mathrm{a}]}$ Giovanni Smaldone, ${ }^{[\mathrm{a}]}$ Pasquale De Antonellis, ${ }^{[\mathrm{b}]}$ Luciano Pirone ${ }^{[\mathrm{c}]}$ \\ MariaNeve Carotenuto, ${ }^{[b]}$ Alessandro Alonzi, ${ }^{[b]}$ Sonia Di Gaetano, ${ }^{[a]}$ Massimo Zollo, ${ }^{[b, d]}$ \\ Emilia M. Pedone, ${ }^{*[a]}$ and Roberto Fattorusso*[e]
}

The human prune protein (h-prune) belongs to the phosphoesterase DHH family. Its over-expression is correlated with cancer progression, tumor aggressiveness and advanced disease status in breast and gastric cancers. ${ }^{[1]}$ The inhibition of the phosphodiesterase activity with dipyridamole suppresses cell motility in breast cancer cell lines. ${ }^{[2]}$ The N-terminus of the h-prune sequence contains the $\mathrm{DHH}$ and DHHA2 domains that are involved in its enzymatic function, related to cAMP pools and inorganic polyphosphates in the cells. ${ }^{[2,3]}$ These domains are then followed by a C-terminal third domain, the involvement of which in the interaction with different partners has been recently proposed. ${ }^{[4-7]}$ The best characterized h-prune partner is Nm23-H1 the interaction of which is mediated through casein kinase (CKI, CKII) phosphorylation of Ser120, Ser122 and Ser125, ${ }^{[5]}$ and the complex promotes enhanced cell motility. ${ }^{[8-10]}$ A threedimensional characterization of the C-terminal h-prune region (residues 354-453), named c-prune, obtained through NMR spectroscopy and molecular dynamics (MD) analysis has been very recently obtained ${ }^{[11]}$ and had revealed the presence of an intrinsically disordered domain (residues 370-453), which nonetheless includes two regions with a

[a] Dr. D. Diana, ${ }^{+}$Dr. G. Smaldone ${ }^{+}{ }^{+}$Dr. S. Di Gaetano,

Dr. E. M. Pedone

Istituto di Biostrutture e Bioimmagini, CNR

via Mezzocannone 16, 80134, Napoli (Italy)

E-mail: empedone@unina.it

[b] Dr. P. De Antonellis, Dr. M. Carotenuto, Dr. A. Alonzi,

Prof. M. Zollo

CEINGE Centro di Ingegneria Genetica e Biotecnologie Avanzate Via Salvatore, 486, 80145, Napoli, (Italy)

[c] Dr. L. Pirone

Istituto di Cristallografia, C.N.R. Via Amendola 122/O, 70126, Bari (Italy)

[d] Prof. M. Zollo

Dipartimento di Medicina Molecolare e Biotecnologie Mediche Università Federico II Via Pansini, 5, 80131, Napoli (Italy)

[e] Prof. R. Fattorusso

Dipartimento di Scienze e Tecnologie Ambientali

Biologiche e Farmaceutiche, Seconda Università di Napoli via Vivaldi, 46, 81100, Caserta (Italy)

E-mail: roberto.fattorusso@unina2.it

$\left.{ }^{+}\right]$These authors contributed equally to this work.

Supporting information for this article is available on the WWW under http://dx.doi.org/10.1002/chem.201302168. degree of helical propensity. In particular, the helix $\alpha 3$ is comprised in a small compact region constrained by a disulfide-bridged cycle.

The NMR mapping of c-prune surface regions involved in the interaction with $\mathrm{Nm} 23-\mathrm{H} 1$ has been also achieved and has shown that the small globular section of c-prune represents part of the Nm23-H1 binding epitope ${ }^{[11]}$ Furthermore, a competitive permeable peptide (CPP) has been developed, which mimics the Nm23-H1 minimal region of interaction (amino acids 115-128) ${ }^{[5]}$ and can bind to c-prune, inhibiting the formation of the Nm23-H1-h-prune complex in vitro and in vivo; consequently it has potential use in therapy of Neuroblastoma. ${ }^{[1]}$ In-cell NMR spectroscopy represents a very promising technique in systems biology, allowing structural and functional study of biological macromolecules in their physiological environment. ${ }^{[12-14]}$ Interestingly, comparable results between analyses performed on whole live cells and extracts have been obtained. ${ }^{[15]}$ These methodologies become more intriguing when the molecules of interest are intrinsically disordered proteins, which constitute a relevant part of the cellular proteome,${ }^{[16]}$ the structural features of which are particularly sensible to the cellular environments. ${ }^{[17]}$

Indeed, elucidating structure-activity relationships of intrinsically disordered proteins (IDPs) in the cellular environment is strongly needed to shed light on the role of poorly folded domains in the cellular metabolism. Herein, we structurally characterize, in human cell lysates, the functional interactions of h-prune ID third domain with its endogenous protein partners, particularly $\mathrm{Nm} 23-\mathrm{H} 1,{ }^{[2]}$ the glycogen synthase kinase- $\beta$ (GSK-3 $\beta$ ), ${ }^{[6]}$ ASAP $^{[18]}$ and gelsolin ${ }^{[7]}$ using fast NMR spectroscopy experiments. The cellular analyses were carried out by adding ${ }^{15} \mathrm{~N}$-labeled c-prune to HEK293 lysates. Despite the high abundance of endogenous, unlabeled proteins, the resulting $2 \mathrm{D}{ }^{15} \mathrm{~N},{ }^{1} \mathrm{H}$ HSQC spectra of cprune in HEK293 lysates were superimposable with the reference spectra of the protein in buffer (Figure 1a and Figure S2 in the Supporting Information) indicating that cprune retains the same conformation in cellular environment, very likely preserving its oxidized form, as indicated by the chemical shift of Cys417 and Cys432. Nonetheless, the average c-prune amide resonance signal intensity in the HEK293 lysate was notably reduced with an overall increase in proton linewidths correlating well with the expected in- 
a)

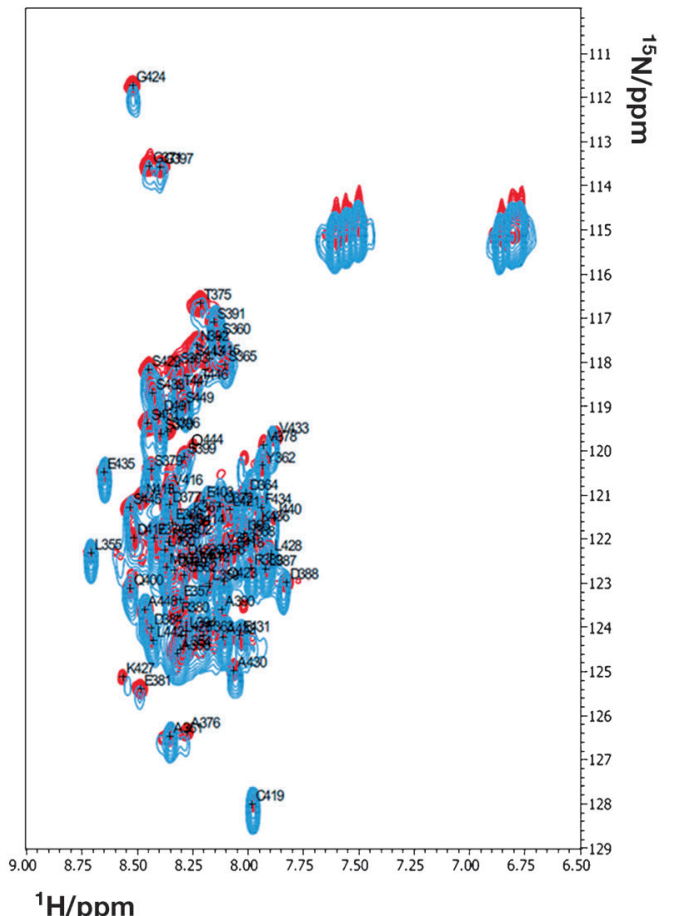

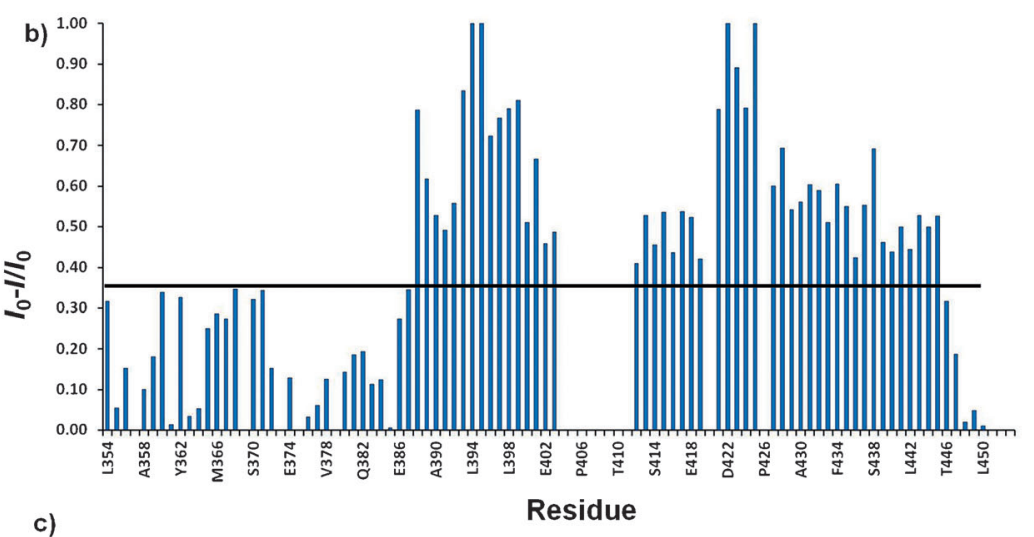

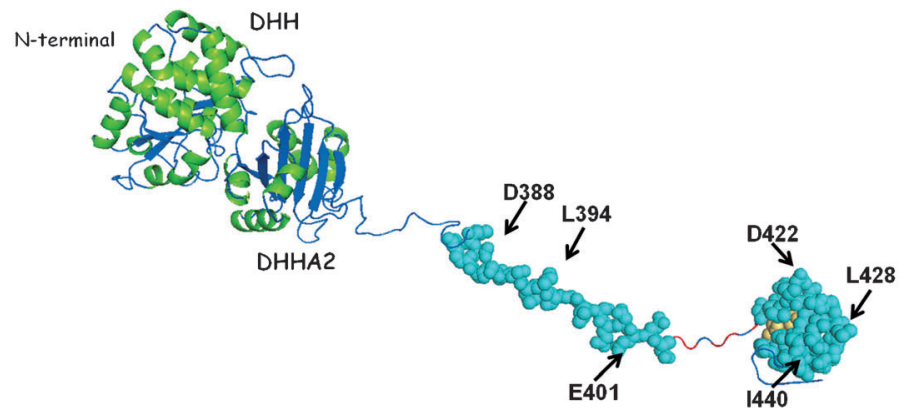

C-terminal

Figure 1. a) Overlay of the 2D ${ }^{15} \mathrm{~N},{ }^{1} \mathrm{H}$ HSQC correlation spectra of purified c-prune in NMR buffer solution (red) and of c-prune in HEK-293 lysate (blue). b) Intensity ratios for all c-prune residues extracted from $2 \mathrm{D}{ }^{15} \mathrm{~N},{ }^{1} \mathrm{H}$ HSQC experiments performed in HEK-293 lysate over-expressing Nm23-H1. c) The amino acids showing large variations upon complex formation with Nm23-H1 $\left(I_{0}-I / I_{0} \geq 0.36\right)$ are mapped in cyan onto the h-prune surface. The region highlighted in red corresponds to the polyproline sequence whereas the region in yellow corresponds to a disulfide bridge between $\mathrm{C} 419$ and C437.

crease in the protein total correlation time $\left(\tau_{c}\right)$ due to cellular viscosity. ${ }^{[19]}$

To investigate the specific association between c-prune and $\mathrm{Nm} 23-\mathrm{H} 1$, a starting solution of purified ${ }^{15} \mathrm{~N}$-labeled cprune was added to HEK293 lysate over-expressing Nm23H1 (Figure S1a in the Supporting Information). As a result, intensities of certain resonances are strongly reduced whereas a small number are slightly shifted, indicating the formation of a high-molecular-weight complex between Nm23-H1 and cprune. The extent of the signal decrease is reported as function of the protein sequence (Figure $1 \mathrm{~b}$ and Figure S3a in the Supporting Information), and the residues most affected by the interaction, mapped onto the c-prune surface (Figure 1c), mostly correspond to those unveiled by the in vitro NMR binding analysis. ${ }^{[11]}$ In particular, in cell lysates NMR analysis outlines two discontinuous regions encompassing amino acids from 388 to 404 and the more compact region constituted by the disulfide-bridged cycle and the $\alpha 3$ helical region (amino acids 412-445). To further validate the specific interaction of c-prune-Nm23-H1, the NMR binding experiments were repeated by using the human neuroblastoma SH-SY5Y lysates naturally expressing high amount of

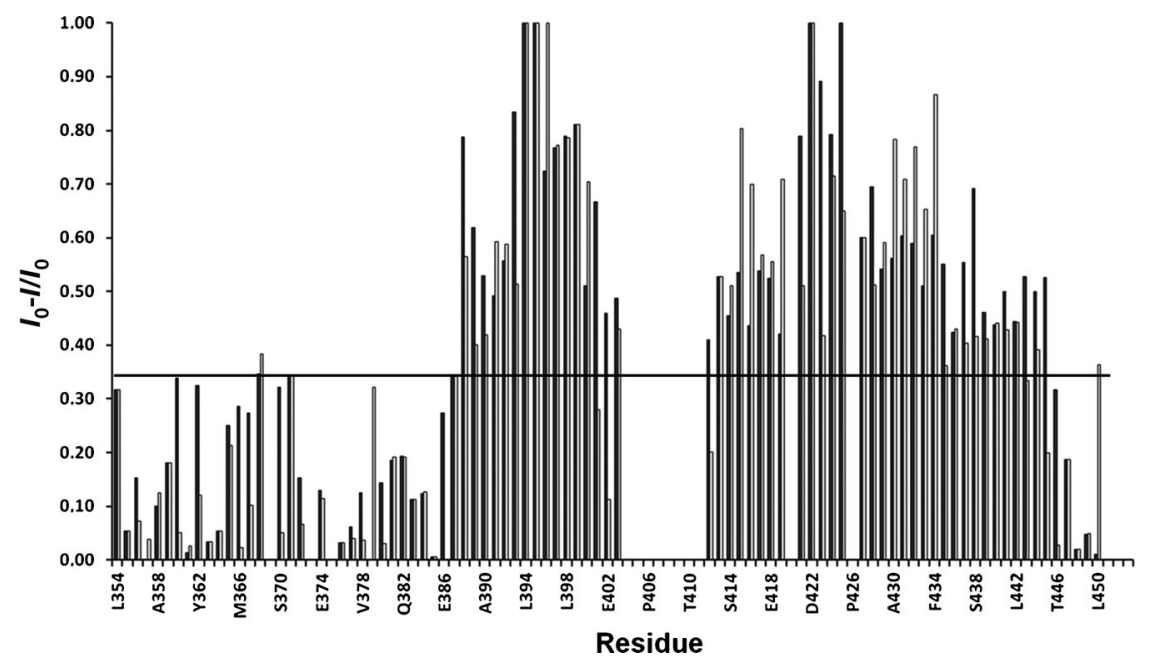

Figure 2. Intensity ratios for all c-prune residues $\left(I_{0}-I / I_{0} \geq 0.36\right)$ extracted from $2 \mathrm{D}{ }^{15} \mathrm{~N},{ }^{1} \mathrm{H}$ HSQC experiments performed in SH-SY5Y lysate (grey) and compared to the data obtained in HEK-293 lysate (black). 
Nm23-H1 (Figures S1a and S3b in the Supporting Information).

As showed in Figure 2, SH-SY5Y extracts confirmed that, upon the complex formation, Nm23-H1 binding epitope corresponds to the two regions identified above. On this basis, we used site-directed mutagenesis to better define the binding site for Nm23-H1. In vitro NMR interaction studies indicated that two negatively charged amino acids, D388 and D422, significantly contribute to the c-prune-Nm23-H1 complex. $^{[11]}$ Accordingly, further functional studies showed that the over-expression of h-prune D388A and D422A mutants did not induce cell migration and that the mutants interacted weakly with Nm23-H1. ${ }^{[1]}$ To correlate the activity loss of the mutants with an in-cell inability to bind their functional partner, we investigated the binding of c-prune D388A or D422A mutants with Nm23-H1. As expected, D388A and D422A show no significant changes in the NMR peak intensities (Figure S4a and $b$ in the Supporting Information), confirming that these two single-point mutations within the c-prune binding epitope compromised its ability to interact with the Nm23-H1 both in vitro and in vivo. Guided by the NMR interaction analysis, we designed another c-prune single point mutation affecting the Nm23$\mathrm{H} 1$ binding site.

In particular, we substituted L394 to alanine to evaluate whether uncharged residues also contribute to the complex formation. NMR binding experiments of the ${ }^{15} \mathrm{~N}$-labeled L394A mutant in HEK293 lysate show features of a remnant, substantially reduced interaction (Figure S4c in the Supporting Information). Although minor chemical shift changes are observed, the pattern of resonances broadening is significantly reduced, mostly including residues of the cprune $\mathrm{C}$ terminus. We conclude that L394, which sits at the center of the c-prune-Nm23-H1 binding site, plays a significant role in the stabilization of c-prune-Nm23-H1 interaction, in vivo. To further validate our system we used cellular lysates transfected with two well characterized single-point mutants of Nm23-H1, P96S and S120G, both of which are known to alter Nm23-H1 biochemical activity or structure and affect the cell motility. ${ }^{[20]}$ To this aim, we added ${ }^{15} \mathrm{~N}$-labeled c-prune to HEK293 extracts over-expressing each Nm23-H1 mutant (Figure S1a in the Supporting Information). The signal intensities of the extracts over-expressing Nm23-H1 S120G or P96S mutants were comparable to those observed in the corresponding extracts not over-expressing endogenous Nm23-H1 (Figures S5 and S6 in the Supporting Information), demonstrating that the loss of activity of Nm23-H1 S120G or P96S mutants depends on their inability to bind c-prune.

Moreover, we extended functional studies in cell lysates to other h-prune endogenous partners, such as GSK3 $\beta$, gelsolin and ASAP1 (Figure S1b in the Supporting Information). Upon addition of HEK293 extracts over-expressing GSK3 $\beta$ or gelsolin to starting solutions of ${ }^{15} \mathrm{~N}$-labeled cprune the NMR peaks weakened, indicating c-prune interaction with each of the proteins. In particular, residues between Q356 and S396 and residues from A361 to R389 of a)

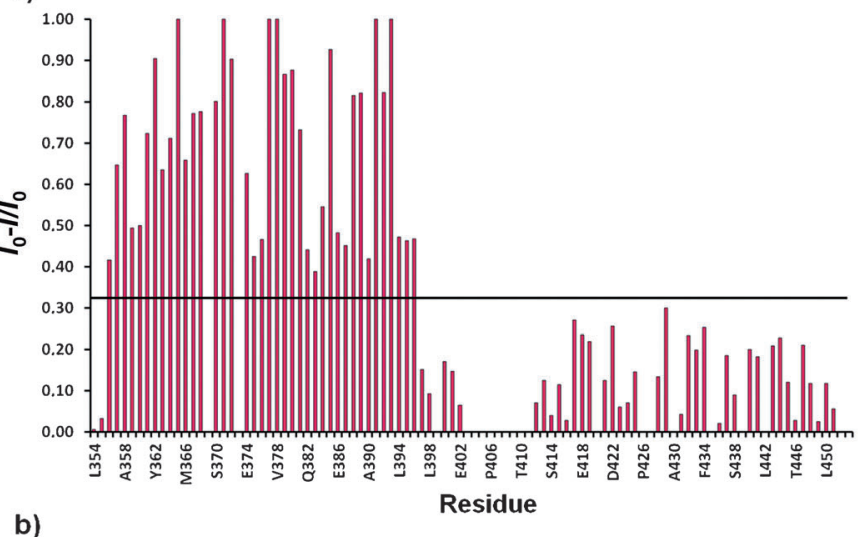

N-terminal
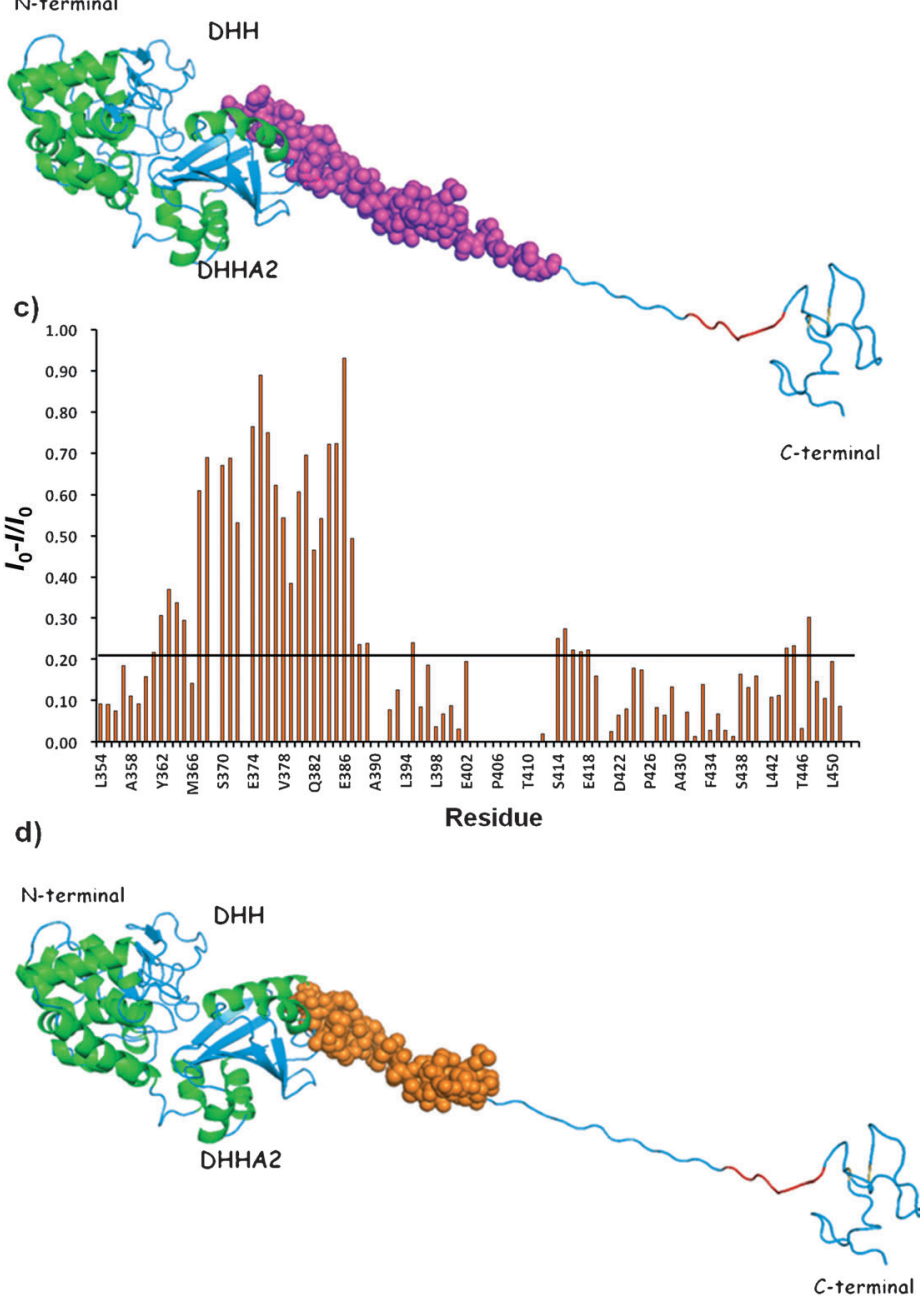

Figure 3. a) Intensity ratios for all c-prune residues $\left(I_{0}-I / I_{0}>0.32\right)$ extracted from $2 \mathrm{D}{ }^{15} \mathrm{~N},{ }^{1} \mathrm{H}$ HSQC experiments performed in HEK-293 lysate over-expressing GSK-3 $\beta$. b) The amino acids showing large variations upon complex formation with GSK-3 $\beta$ are mapped in magenta onto the h-prune surface. c) Intensity ratios for all c-prune residues $\left(I_{0}-I / I_{0} \geq 0.21\right)$ extracted from 2D ${ }^{15} \mathrm{~N},{ }^{1} \mathrm{H}$ HSQC experiments performed in HEK-293 lysate over-expressing gelsolin. d) The amino acids showing large variations upon complex formation with gelsolin are mapped in orange onto the h-prune surface. The region on h-prune in red corresponds to the polyproline sequence whereas the region in yellow corresponds to a disulfide bridge between Cys419 and Cys437. 
the ${ }^{15} \mathrm{~N}$ labeled c-prune show an extensive line broadening in the presence of HEK293 lysate over-expressing GSK3 $\beta$ and gelsolin, respectively (Figure 3). Interestingly, in cell lysate NMR spectroscopy experiments outline GSK3 $\beta$ and gelsolin binding sites that lie on the $\mathrm{N}$-terminal portion of cprune, indicating a remarkable binding versatility of this domain, likely due mostly to its intrinsically disordered nature. Indeed, residues 353-365 of c-prune constitute the C-terminal region of h-prune DHHA2 domain; thus GSK3b and gelsolin interaction also with this domain cannot be excluded. On the other hand, in the presence of cellular extracts over-expressing ASAP1 no changes in signal intensities in the c-prune ${ }^{15} \mathrm{~N},{ }^{1} \mathrm{H}$ HSQC spectrum were observed corroborating the hypothesis that this partner does not interact with the C-terminal domain of h-prune but, probably, with the other two domains, DHH and DHHA2, involved in the catalytic activity, and is therefore able to stimulate $h$ prune exopolyphosphatase and cAMP-PDE previously characterized activities. ${ }^{[3]}$

In conclusion, we show, in human cell lysates, how the Cterminal domain of h-prune functional activity is mediated by appropriate interactions with endogenous partners. The NMR analysis shows that the intrinsically disordered third domain of h-prune retains its conformational preferences in a cellular environment, at the same time showing a valuable capacity to recognize different cellular partners by means of appropriate protein portions. Detecting interaction in lysates represents a very useful tool for studying protein-protein interactions because many proteins are difficult to express in E. coli, to purify or to obtain with certain post-translational modifications. Overall, this study shows that protein functions mediated by protein-protein interactions can be accurately followed in cell lysates with NMR spectroscopy fast experiments, which could be easily used for a very efficient NMR drug discovery strategy, either starting from fragments or developed ligands. ${ }^{[21-24]}$ Finally, this functional characterization contributes to a better understanding of the oncogenic h-prune biological activity, providing the basis for developing new drugs in cancer therapy.

\section{Acknowledgements}

This work was funded by the Italian MIUR financial support FIRB RBAP114AMK_006, FIRB RBFR12WB3W_002 and PRIN 2010 2010M2JARJ_002. G.S. was supported by Dottorato in Scienze Chimiche. Associazione Italiana Ricerca sul cancro AIRC 2012 (M.Z.), FP7-Tumic HEALTH-F2-2008-201662 (M.Z.), PRIN (E5AZ5F) 2008 (M.Z.), M.C. was supported by Dottorato in Biologia Computazionale e Bioinformatica, Università Federico II of Naples. We thank Leopoldo Zona for excellent technical assistance.

Keywords: human cell lysates • intrinsically disordered proteins $\cdot$ NMR spectroscopy $\cdot$ protein-protein interactions • structure elucidation
[1] M. Zollo, A. Andrè, A. Cossu, M. C. Sini, A. D’Angelo, N. Marino, M. Budroni, F. Tanda, G. Arrigoni, G. Palmieri, Clin. Cancer Res. 2005, 11, 199-205.

[2] A. D’Angelo, L. Garzia, A. André, P. Carotenuto, V. Aglio, O. Guardiola, G. Arrigoni, A. Cossu, G. Palmieri, L. Aravind, M. Zollo, Cancer Cell 2004, 5, 137-149.

[3] M. Tammenkoski, K. Koivula, E. Cusanelli, M. Zollo, C. Steegborn, A. A. Baykov, R. Lahti, Biochemistry 2008, 47, 9707-9713.

[4] S. Middelhaufe, L. Garzia, U. M. Ohndorf, B. Kachholz, M. Zollo, C. Steegborn, Biochem. J. 2007, 407, 199-205.

[5] L. Garzia, A. D’Angelo, A. Amoresano, S. K. Knauer, C. Cirulli, C. Campanella, R. H. Stauber, C. Steegborn, A. Iolascon, M. Zollo, Oncogene 2008, 27, 1853-1864.

[6] T. Kobayashi, S. Hino, N. Oue, T. Asahara, M. Zollo, W. Yasui, A. Kikuchi, Mol. Cell. Biol. 2006, 26, 898-911.

[7] L. Garzia, C. Roma, N. Tata, D. Pagnozzi, P. Pucci, M. Zollo, J. Bioenerg. Biomembr. 2006, 38, 205-213.

[8] A. Forus, A. D'Angelo, J. Henriksen, G. Merla, G. M. Maelandsmo, V. A. Florenes, S. Olivieri, B. Bjerkehagen, L. A. Meza-Zepeda, F. del Vecchio Blanco, C. Müller, F. Sanvito, J. Kononen, J. M. Nesland, $\emptyset$. Fodstad, A. Reymond, O. P. Kallioniemi, G. Arrigoni, A. Ballabio, O. Myklebost, M. Zollo, Oncogene 2001, 20, 6881-6890.

[9] A. Reymond, S. Volorio, G. Merla, M. Al-Maghtheh, O. Zuffardi, A. Bulfone, A. Ballabio, M. Zollo, Oncogene 1999, 18, 7244-7252.

[10] P. S. Steeg, M. Zollo, T. Wieland, Naunyn Schmiedebergs Arch. Pharmacol. 2011, 384, 331-339.

[11] M. Carotenuto, E. Pedone, D. Diana, P. de Antonellis, S. Džeroski, N. Marino, L. Navas, V. Di Dato, M. N. Scoppettuolo, F. Cimmino, S. Correale, L. Pirone, S. M. Monti, E. Bruder, B. Zenko, I. Slavkov, F. Pastorino, M. Ponzoni, J. H. Schulte, A. Schramm, A. Eggert, F. Westermann, G. Arrigoni, B. Accordi, G. Basso, M. Saviano, R. Fattorusso, M. Zollo, Sci. Rep. 2013; DOI: 10.1038/srep01351.

[12] Y. Ito, P. Selenko, Curr. Opin. Struct. Biol. 2010, 20, 640-648.

[13] S. Reckel, F. Löhr, V. Dötsch, ChemBioChem 2005, 6, 1601-1606.

[14] Z. Serber, A. T. Keatinge-Clay, A. E. Kelly, S. M. Miller, V. Dötsch, J. Am. Chem. Soc. 2001, 123, 2446-2447.

[15] P. Selenko, G. Wagner, J. Struct. Biol. 2007, 158, 244-253.

[16] D. Kovacs, B. Szabo, R. Pancsa, P. Tompa, Arch. Biochem. Biophys. 2013, 531, 89-90.

[17] P. Tompa, Nat. Chem. Biol. 2012, 8, 597-600.

[18] T. Müller, U. Stein, A. Poletti, L. Garzia, M. Rothley, D. Plaumann, W. Thiele, M. Bauer, A. Galasso, P. Schlag, M. Pankratz, M. Zollo, J. P. Sleeman, Oncogene 2010, 29, 2393-2403.

[19] P. Selenko, Z. Serber, B. Gadea, J. Ruderman, G. Wagner, Proc Natl. Acad. Sci. USA 2006, 103, 11904-11909.

[20] Y. I. Kim, S. Park, D. I. Jeoung, H. Lee, Biochem. Biophys. Res. Commun. 2003, 307, 281-289.

[21] M. Pellecchia, B. Becattini, K. J. Crowell, R. Fattorusso, M. Forino, M. Fragai, D. Jung, T. Mustelin, L. Tautz, Expert Opin. Ther. Targets 2004, 8, 597-561.

[22] R. Fattorusso, D. Jung, K. J. Crowell, M. Forino, M. Pellecchia, J. Med. Chem. 2005, 48, 1649-1656.

[23] R. Fattorusso, S. Frutos, X. Sun, N. J. Sucher, M. Pellecchia, Phytomedicine 2006, 13, 16-22.

[24] M. Pellecchia, I. Bertini, D. Cowburn, C. Dalvit, E. Giralt, W. Jahnke, T. L. James, S. W. Homans, H. Kessler, C. Luchinat, B. Meyer, H. Oschkinat, J. Peng, H. Schwalbe, G. Siegal, Nat. Rev. Drug Discovery 2008, 7, 738-745.

Received: June 6, 2013 Published online: August 12, 2013 